\title{
Hábitos de lectura en inglés de maestros \\ bilingües en formación ${ }^{*}$
}

\section{English Reading Habits Hábitos de leitura em of Pre-Service English inglês de professores Teachers bilíngues em formação}

Sindy Patricia Cardona Puello** https://orcid.org/0000-0002-9528-4266

Alexander Javier Osorio Beleño*** https://orcid.org/0000-0002-4740-8527

Amalfi de la Cruz Herrera Valdez**** https://orcid.org/0000-0002-7600-6842

Sonia Isabel Bedoya Soto*** https://orcid.org/0000-0001-8453-2407

\section{Para citar este artículo}

Cardona-Puello, S. P., Osorio-Beleño, A. J., Herrera-Valdez, A. de la C. y Bedoya-Soto, S. I. (2022). La lectura del libro álbum como mediación hacia el desarrollo socioemocional. Folios, (55).

Este artículo muestra parte de los resultados del proyecto de investigación titulado Análisis del hábito y la comprensión lectora en inglés de los estudiantes del nivel de formación básico profesional de la Licenciatura en Bilingüismo con énfasis en Inglés de Unicolombo.

* Magíster en Estudios Latinoamericanos con orientación en Cultura y Comunicación. Fundación Universitaria Colombo Internacional-Unicolombo. Licenciatura en Bilingüismo con énfasis en inglés.

Correo: scardona@unicolombo.edu.co

*** Magíster en Educación. Fundación Universitaria Colombo Internacional-Unicolombo. Licenciatura en Bilingüismo con énfasis en inglés.

Correo: aosorio@unicolombo.edu.co

**** Magíster en Educación. Fundación Universitaria Colombo Internacional-Unicolombo. Licenciatura en Bilingüismo con énfasis en inglés.

Correo: aherrera@unicolombo.edu.co

${ }^{* * * * *}$ Magíster en Educación. Fundación Universitaria Colombo Internacional-Unicolombo. Licenciatura en Bilingüismo con énfasis en inglés.

Correo: sbedoya@unicolombo.edu.co

\section{https://doi.org/10.17227/folios.55-12167}

Artículo recibido

$27 \cdot 07 \cdot 2020$

Artículo aprobado

$02 \cdot 07 \cdot 2021$ 


\section{Resumen}

Este artículo muestra los resultados de una investigación realizada en una institución universitaria de Cartagena (Colombia) con el fin de analizar los hábitos de lectura en inglés de los estudiantes de semestres avanzados de un programa de Licenciatura en Bilingüismo. En dicha investigación se recurrió a técnicas de recolección de datos cuantitativos y cualitativos (enfoque mixto) para determinar cómo es el hábito lector en inglés de los estudiantes de dicho programa. Según los resultados obtenidos, la mayoría de los estudiantes $(78,6 \%)$ son lectores ocasionales y tan solo el 9,2 \% son lectores frecuentes; además, la lectura suele estar asociada a obligaciones de tipo académico más que a una motivación intrínseca hacia la lectura en lengua extranjera.

\section{Palabras clave}

hábitos de lectura; aprendizaje del inglés; alfabetización académica; educación superior

\section{Abstract}

This paper shows the results of a research carried out at a university institution in Cartagena (Colombia), to analyze the reading habits in English of students in advanced semesters of a Bachelor's Degree in Bilingualism program. In this research, we used quantitative and qualitative data collection techniques (mixed approach) to determine what the reading habits of students in this program are like in English. According to the results, most of the students (78,6 \%) are occasional readers, and only $9,2 \%$ of them are frequent readers. Furthermore, reading is associated with academic obligations rather than an intrinsic motivation towards reading in a foreign language.

\section{Keywords}

reading habits; learning English; academic literacy; higher education

\section{Resumo}

Este artigo apresenta os resultados da pesquisa realizada em uma instituição universitária de Cartagena (Colômbia), com o objetivo de analisar os hábitos de leitura em inglês dos alunos do semestre avançado do curso de Bacharelado em Bilinguismo. Nesta pesquisa, recorreu-se a técnicas quantitativas e qualitativas de coleta de dados (abordagem mista) para determinar como é o hábito da leitura em inglês dos alunos deste programa. De acordo com os resultados obtidos, a maioria dos estudantes (78,6 \%) são leitores ocasionais e apenas 9,2 \% são leitores frequentes; Além disso, a leitura é geralmente associada a obrigações acadêmicas, e não a uma motivação intrínseca à leitura em uma língua estrangeira.

\section{Palavras chave}

hábitos de leitura; aprendizado de inglês; alfabetização acadêmica; ensino superior 


\section{Introducción}

El Estado colombiano ha creado distintos programas para el fomento del bilingüismo en el país, partiendo de la premisa de que los estudiantes deben estar preparados para enfrentar los retos de un mundo globalizado. Uno de los programas más representativos fue el Plan Nacional de Bilingüismo (PNB) 2004-2019, dentro del cual se diseñaron los Estándares Básicos de Competencias en Lenguas Extranjeras: Inglés para fortalecer las competencias comunicativas en dicho idioma siguiendo los criterios del Marco Común Europeo de Referencia para las Lenguas (MCER). Este programa estableció que los profesionales de todas las carreras en el país debían egresar de la universidad con un nivel de inglés B2, mientras que los egresados de programas de licenciatura en idiomas o inglés debían manejar un nivel mínimo de C1. Para alcanzar dicho nivel, el PNB desarrolló el Proyecto para el Fortalecimiento a las Licenciaturas en Idiomas/ Inglés, con el objetivo de "realizar un diagnóstico general de la situación académica de los programas y proponer un plan de mejoramiento para fortalecer las licenciaturas en idiomas de las universidades en Colombia" (Cárdenas, 2018, p. 135).

Posteriormente, el Ministerio de Educación Nacional estableció mediante la Resolución 18583 de 2017, por la cual se ajustan las características específicas de calidad de los programas de licenciatura en Colombia, que todas las licenciaturas en Español e Inglés, Lenguas Modernas, Lenguas Extranjeras o en Bilingüismo con énfasis en una lengua extranjera deberán evidenciar que sus estudiantes han obtenido el nivel $\mathrm{C} 1$ en la lengua de énfasis.

Para obtener dicho nivel, el hablante debe ser capaz, entre otras cosas, de comprender textos extensos y de distintos grados de complejidad, así como de producir textos bien estructurados que den cuenta de una corrección lingüística y de una pertinencia discursiva. Es aquí donde el hábito lector en inglés cobra relevancia, puesto que la lectura frecuente y motivada en el segundo idioma puede contribuir al conocimiento adecuado de este. Iftanti (2012) entiende el hábito de lectura en inglés como el deseo de leer en inglés de manera reiterada, lo que lo convierte en un comportamiento permanente e intencional. Es decir, un estudiante con hábito lector en inglés es aquel que adopta la lectura como parte de sus actividades diarias y la incorpora a su propia naturaleza como estudiante de lengua extranjera. Dicho hábito estaría conformado, según esta autora, por distintos aspectos, tales como: fluidez, automatismo, exactitud, prácticas de lectura altamente disfrutables, gran cantidad de lecturas, buen nivel 
de inglés, actitud positiva frente a la lectura, lectura ávida y consciente, elección propia de libros, afán por recibir libros como obsequio y lectura regular después de la jornada escolar.
La formación del hábito lector se ve influenciada por varios factores de tipo textual, personal y ambiental, tal como lo evidenció el Ministerio de Educación, Cultura y Deporte en España (2001) al realizar un estudio sobre el hábito de lectura de los adolescentes (véase la tabla 1).

Table 1. Factores incidentes en los hábitos lectores

\begin{tabular}{|c|c|c|}
\hline Factores textuales & Factores personales & Factores ambientales \\
\hline $\begin{array}{ll}\text { - } & \text { Interés del material } \\
\text { - } & \text { Tipo de publicación } \\
\text { - } & \text { Tipo de material de lectura } \\
\text { - } & \text { Tipo de cubierta } \\
\text { - } & \text { Autor } \\
\text { - } & \text { Título } \\
\text { - } & \text { Publicidad } \\
\text { - } & \text { Características de los personajes }\end{array}$ & $\begin{array}{ll}\text { - } & \text { Sexo } \\
\text { - } & \text { Edad } \\
\text { - } & \text { Nivel de estudios } \\
\text { - } & \text { Competencia lectora } \\
\text { - } & \text { Clase social } \\
\text { - } & \text { Disponibilidad de tiempo libre } \\
\text { - } & \text { Actitud hacia la lectura }\end{array}$ & $\begin{array}{ll}\text { - } & \text { Entorno familiar } \\
\text { - } & \text { Escuela } \\
\text { - } & \text { Bibliotecas públicas } \\
\text { - } & \text { Grupo de iguales } \\
\text { - } & \text { Autores, ilustradores, } \\
\text { - } & \text { editores y libreros } \\
& \text { dedios tecnológicos y } \\
\text { - } & \text { Oferta cultural } \\
\text { - } & \text { Sociedad en general }\end{array}$ \\
\hline
\end{tabular}

Fuente: Ministerio de Educación, Cultura y Deporte de España (2001, p. 39).

Por otra parte, son varios los autores que han hecho una clasificación de los lectores según el hábito. Fatiloro et al. (2017), por ejemplo, consideran que los lectores se pueden clasificar en cuatro tipos principales:

heavy readers (reading various texts and novels as much as possible and as many times as possible in a month); moderate readers (reading few texts and novels as seem convenient for them); dormant readers (those who liked to read and they regarded themselves readers but did not take time to read regularly and update their knowledge). These readers give priority to other social activities such as sports, partying and school work. Unmotivated non readers (those who do not have any inclination to read neither are they open to the suggestion of future reading nor do they have any positive attitude towards people who read). (p. 107)

Cultivar un buen hábito de lectura en inglés llevará a los estudiantes universitarios, especialmente a los futuros docentes de este idioma, a manejarlo con maestría y fluidez. Sin embargo, tal como han evidenciado algunos trabajos, no siempre los estudiantes responden a dicho nivel de exigencia, puesto que su aproximación a la lectura en inglés es escasa. En su investigación sobre hábitos de lectura en inglés de estudiantes de programas de educación en España, Gómez (2014) encontró que el 63,6 \% de los futuros maestros manifestaron que solo leen materiales obligatorios para poder aprobar las asignaturas, lo cual revela cierta renuencia hacia la lectura académica en inglés. En este contexto, los programas de lenguas extranjeras deben revisar periódicamente sus planes de estudio con miras a fortalecer la formación bilingüe, lo que conlleva la necesidad de realizar estudios en torno a las prácticas lectoras en inglés que se llevan a cabo dentro y fuera del aula para vislumbrar posibles planes que garanticen un mejor desempeño comunicativo en dicha lengua.

En la conformación de estrategias para el mejoramiento de la competencia lectora en el segundo idioma de los futuros maestros bilingües es crucial realizar previamente un diagnóstico del hábito 
lector de los estudiantes, así como de los factores que inciden sobre él. En relación con lo anterior, este artículo muestra los resultados de una investigación realizada en el programa de Licenciatura en Bilingüismo con énfasis en Inglés de una institución universitaria bilingüe en Cartagena (Colombia), cuyo objetivo fue determinar cuáles son los hábitos de lectura en inglés de los estudiantes de semestres avanzados de dicho programa. Conocer las prácticas lectoras en inglés de los estudiantes permitirá pensar en acciones pedagógicas necesarias para propiciar un acercamiento más significativo hacia la lectura en lengua inglesa y el mejoramiento de su desempeño en la lengua extranjera.

\section{Metodología}

Los fenómenos relacionados con los hábitos de lectura suelen ser multifactoriales y requieren de un análisis que no solo tome en cuenta datos de tipo cuantitativo, sino también de tipo cualitativo. No se trata únicamente de saber si los estudiantes leen o no y con qué frecuencia lo hacen, sino de explorar más a fondo las razones que tienen los individuos para leer, conocer las preferencias y los factores actitudinales que delinean el hábito. Justamente por ello, este trabajo fue realizado bajo un enfoque mixto de investigación, que ayudara a describir las características de un fenómeno en particular (el hábito lector en inglés) para poder tener un perfil detallado de la población estudiada y vislumbrar planes de acción que ayuden a afianzar esa actividad entre los estudiantes.

\section{Población}

El plan de estudios del programa de Licenciatura en Bilingüismo con énfasis en Inglés se estructura en tres niveles de formación: Básico (semestres I-III), básico profesional (semestres IV-VI) y profesional (semestres VII-XIX). El primero de ellos está conformado por un conjunto de asignaturas que aseguran la adquisición de los conocimientos generales en las áreas de pedagogía y de las competencias comunicativas básicas en lengua materna, lengua inglesa y pensamiento lógico-matemático. A partir del segundo nivel de formación, los estudiantes adquieren los saberes específicos o el conocimiento de las teorías, metodologías y debates de carácter disciplinar. Se estudian las principales teorías sobre enseñanza, formación y evaluación; se afianzan las competencias comunicativas en inglés mediante el fortalecimiento de la lectura y escritura académicas en lengua inglesa, y se abordan las problemáticas relacionadas específicamente con la enseñanza del idioma. A partir de esta etapa, los estudiantes cursan todas las asignaturas en inglés, lo que implica un mayor grado de exigencia en las actividades académicas y en las capacidades lectoras en lengua extranjera para tener una adecuada formación disciplinar.

A partir de IV semestre es crucial mantener un buen hábito lector en la lengua meta, de allí surge el interés por trabajar con esta población. En el estudio participaron los 131 estudiantes de los niveles de formación básico profesional y profesional, ${ }^{1}$ es decir, los que cursan de IV a viII semestre. El 74,8 \% de los participantes son mujeres y el $25,2 \%$ son hombres, con un promedio de edad de 23 años.

\section{Instrumentos de recolección de datos y procedimiento}

Para caracterizar los hábitos de lectura en inglés de los estudiantes se aplicó un test de autorreporte diseñado por el equipo de investigación a los 131 estudiantes que cursan las asignaturas de los niveles de formación mencionados (véase el anexo). Dicho test consta de 45 ítems distribuidos en cuatro dimensiones principales: actitud hacia la lectura, frecuencia, motivación y autopercepción. El test fue sometido a validez de contenido por dos especialistas en Lenguas Extranjeras y un lingüista para verificar la pertinencia y claridad en la redacción de cada uno de los reactivos. Además, se sometió a las pruebas psicométricas necesarias para determinar su confiabilidad y validez. En primer lugar, se calculó el alfa de Cronbach de cada una de las dimensiones, y se obtuvieron los siguientes valores:

1 Cabe aclarar que no se contó con estudiantes de ix semestre, puesto que, durante la investigación (segundo periodo del 2019 y primer periodo de 2020) el nuevo plan de estudios aún no había avanzado hasta ese semestre. 
Universidad Pedagógica Nacional

Facultad de Humanidades

actitud: 0,809; frecuencia: 0,794; motivación: 0,832 y autopercepción: 0,705. Luego, se aplicaron técnicas de reducción de datos y se verificaron las condiciones previas para el análisis factorial exploratorio
(AFE) a través de la medida (Kaiser-Meyer-Olkin) KMO. Dicho análisis permitió identificar los factores internos de cada dimensión, tal como se muestra en la tabla 2.

Table 2. Categorización de las dimensiones del test de autorreporte

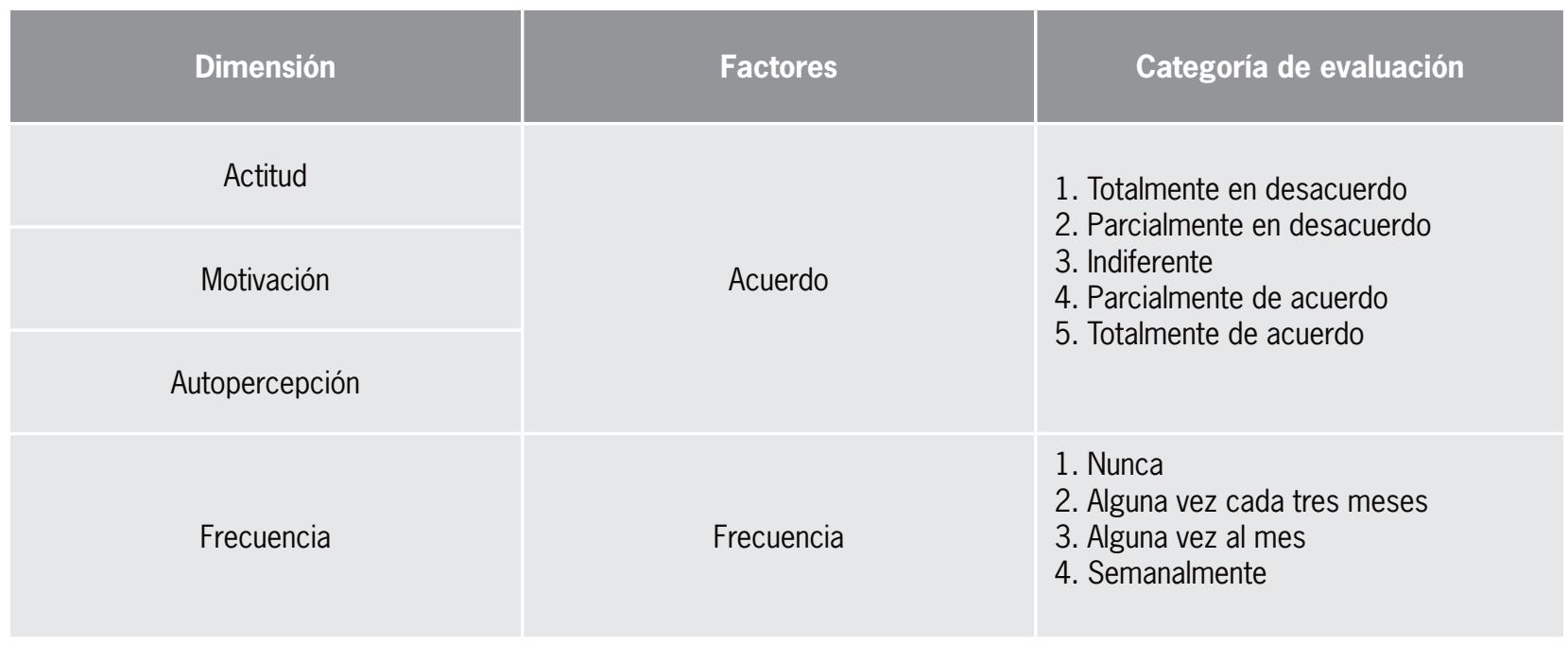

Fuente: elaboración propia 
Como modo de respuesta a los ítems se utilizó una escala tipo likert en cada una de las dimensiones, como se muestra en la tabla 3.

Table 3. Escala de respuesta del test de autorreporte

\begin{tabular}{|c|c|c|}
\hline Dimensión & Tipo de escala & Opciones de respuesta \\
\hline Actitud hacia la lectura & $\begin{array}{c}\text { Gusto por la lectura } \\
\text { Utilidad de la lectura } \\
\text { Disposición } \\
\text { Propósito }\end{array}$ & $\begin{array}{l}\text { Actitud positiva } \\
\text { Actitud negativa }\end{array}$ \\
\hline Frecuencia & $\begin{array}{c}\text { Textos académicos } \\
\text { Textos no académicos }\end{array}$ & $\begin{array}{c}\text { No lector } \\
\text { Lector ocasional } \\
\text { Lector frecuente }\end{array}$ \\
\hline Motivación & $\begin{array}{c}\text { Iniciativa } \\
\text { Necesidad de la lectura }\end{array}$ & $\begin{array}{l}\text { Motivación intrínseca } \\
\text { Motivación extrínseca }\end{array}$ \\
\hline Autopercepción & $\begin{array}{c}\text { Fluidez } \\
\text { Capacidad lectora }\end{array}$ & $\begin{array}{l}\text { Lector no competente } \\
\text { lector principiante } \\
\text { lector competente }\end{array}$ \\
\hline
\end{tabular}

Fuente: elaboración propia 
Para categorizar a los lectores del programa estudiado, se acudió a la clasificación de los lectores de Fatiloro et al. (2017) mencionada en el apartado anterior. Dicha clasificación se articuló con las dimensiones establecidas en el test de autorreporte.

Table 4. Caracterización de los lectores según el hábito

\begin{tabular}{|c|c|c|c|c|}
\hline Dimensión & Heavy Readers & Moderate Readers & Dormant Readers & $\begin{array}{c}\text { Unmotivated } \\
\text { Non Readers }\end{array}$ \\
\hline $\begin{array}{c}\text { Actitud hacia } \\
\text { la lectura }\end{array}$ & Actitud positiva & Actitud positiva & Actitud positiva & Actitud negativa \\
\hline Frecuencia & Lector frecuente & Lector ocasional & Lector ocasional & No lector \\
\hline Motivación & Motivación intrínseca & Motivación intrínseca & $\begin{array}{c}\text { Motivación } \\
\text { extrínseca }\end{array}$ & $\begin{array}{c}\text { Motivación extrínse- } \\
\text { ca/ sin motivación }\end{array}$ \\
\hline
\end{tabular}

Fuente: elaboración propia

Luego, se usó la técnica de grupo focal para dialogar con los estudiantes, a partir de un conjunto de preguntas problematizadoras, para conocer sus apreciaciones acerca de su propio hábito lector en inglés, así como de los factores que ellos consideran influyentes en su actividad lectora. Se realizó un primer grupo focal en la jornada nocturna con estudiantes de IV a viII semestre, y un segundo grupo en la jornada diurna. En cada grupo focal participaron 13 estudiantes de manera voluntaria y fueron moderados por dos integrantes del equipo de investigación. El número de estudiantes en cada grupo focal se determinó siguiendo la literatura consultada, según la cual se establece, dependiendo del tema y objetivo de la investigación, un rango entre seis y doce participantes para que esta técnica cualitativa sea viable. 
Table 5. Diálogo con grupo focal

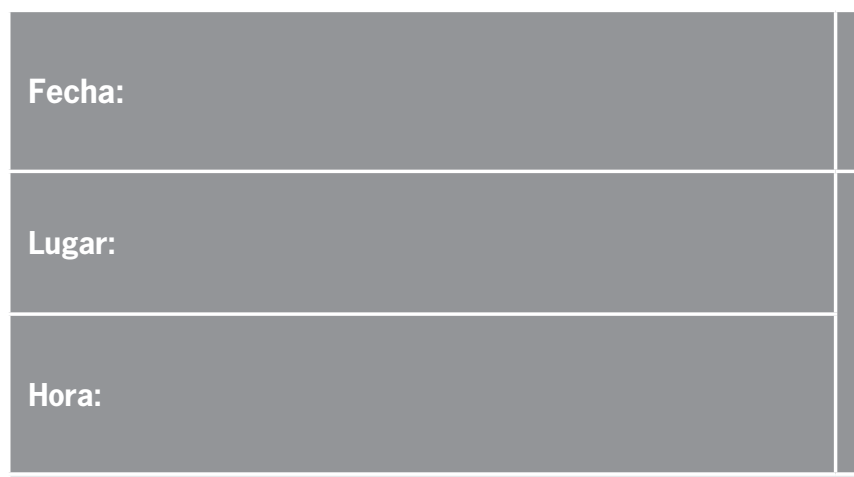

$N{ }^{\circ}$ de participantes:

mujeres ()

hombres ()

1. ¿En general, les gusta leer?

2. ¿Prefieren leer en inglés antes que realizar otras actividades en su tiempo libre?

3. ¿Hay personas de su entorno familiar y social que los animan a leer? ¿Quiénes?

4. ¿Hay personas de su entorno familiar y social que los animan a leer en inglés? ¿Quiénes?

5. ¿Suelen tener tus propios libros de inglés en casa?

6. ¿Suelen estar atentos a las publicaciones más recientes en tu área de estudio (nuevos artículos, lanzamiento de libros, etc.)?

7. ¿No les gusta leer en inglés? ¿Por qué?

8. ¿Cuántos textos académicos en inglés (libros, capítulos de libro, artículos científicos, etc.)

han leído en el último año por iniciativa propia? ¿Podrían mencionar los títulos?

9. ¿Cuántos textos no académicos en inglés (novelas, cuentos, cómics, blogs, revistas de entretenimiento, libros de autoayuda, etc.) han leído en el último año por iniciativa propia? ¿Podrían mencionar los títulos?

10. ¿Les gusta que les obsequien libros en inglés?

11. ¿Consideran que su hábito lector en inglés es producto de la formación académica recibida en el programa?

12. ¿Consideran que el currículo del programa de Licenciatura en Bilingüismo fomenta el hábito de lectura en inglés entre sus estudiantes?

13. ¿De qué manera creen que el programa puede ayudar a fomentar el gusto por la lectura en inglés entre sus estudiantes? 


\section{Resultados}

$\mathrm{Al}$ medir las actitudes hacia la lectura en inglés se obtuvo que la mayoría de ellos $(90,8 \%)$ respondió positivamente a las preguntas relacionadas con la utilidad de la lectura en inglés; es decir, los estudiantes consideran que la lectura en inglés es útil en el proceso de aprendizaje, en la formación personal y en el ejercicio de su profesión. No obstante, al indagar específicamente por el gusto, la mayoría (54,2 \%) mostró una actitud negativa, es decir, que para ellos la lectura en inglés no representa una actividad placentera o no les atrae leer en inglés cualquier tipo de material.
De igual modo, un 38,2 \% respondió estar totalmente de acuerdo con la afirmación "leer en inglés me agota fácilmente" y un 46,6 \% respondió estar totalmente de acuerdo con la afirmación "siento estrés cuando leo en inglés". Esto da cuenta de que la disposición frente a la lectura en inglés de los estudiantes de semestres avanzados del programa de Licenciatura en Bilingüismo no es completamente positiva; como se ve en la gráfica 1, el 44,3\% de los estudiantes presenta una disposición negativa hacia la lectura en inglés.

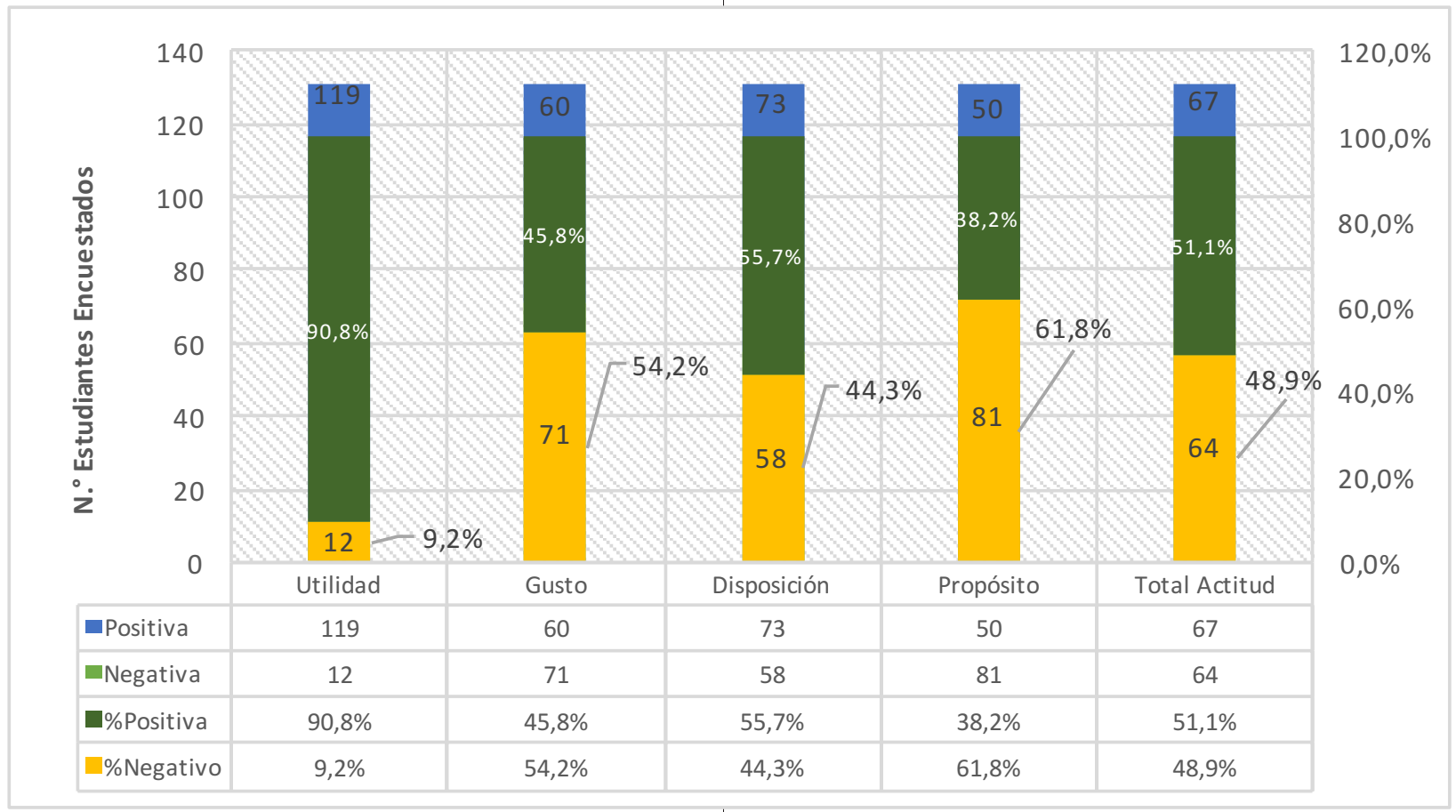

Gráfica 1. Resultados dimensión de actitud 
Ahora bien, en estudios realizados en distintos lugares del mundo se han obtenido resultados similares. En una investigación realizada con estudiantes universitarios de Arabia Saudita, Rajab y Al-Sadi (2015) encontraron que la mayoría de los estudiantes de inglés como lengua extranjera tiene una actitud positiva acerca de la importancia de la lectura en dicho idioma, ya que 207 de los 330 estudiantes participantes están de acuerdo en que la lectura en inglés ayuda a mejorar su nivel en este idioma; no obstante, solo 20 de ellos manifestaron haber leído alguna historia o libro en inglés. Al Nazhari et al. (2016), por su parte, encontraron que el 80,2 $\%$ de los estudiantes participantes en su investigación (English Study Program of Riau University, Indonesia), son conscientes de la importancia de la lectura en inglés para su futuro, pero solo el $25 \%$ de ellos lee materiales en ese idioma con frecuencia. en la gráfica 2 que un 7,6\% se considera como no lector de textos académicos ya que nunca o casi nunca leen este tipo de textos en inglés en su tiempo libre. El 77,10 \% clasifica como lector ocasional de textos académicos, es decir, que leen estos textos en inglés alguna vez al mes o alguna vez cada tres meses. Solo un 15,27 \% de los estudiantes clasifica como lector frecuente de textos académicos, esto es, que lee este tipo de textos semanalmente o a diario. La tendencia es similar cuando se trata de los textos no académicos, ya que el 20,61 \% son no lectores y solo el 16,03 \% de los estudiantes son lectores frecuentes de textos no académicos en blogs, páginas web, revistas de entretenimiento, cómics, material literario, entre otros. La mayoría de los estudiantes encuestados son lectores ocasionales de textos no académicos.

Siguiendo con el presente estudio, en relación con la frecuencia de lectura en inglés, se observa

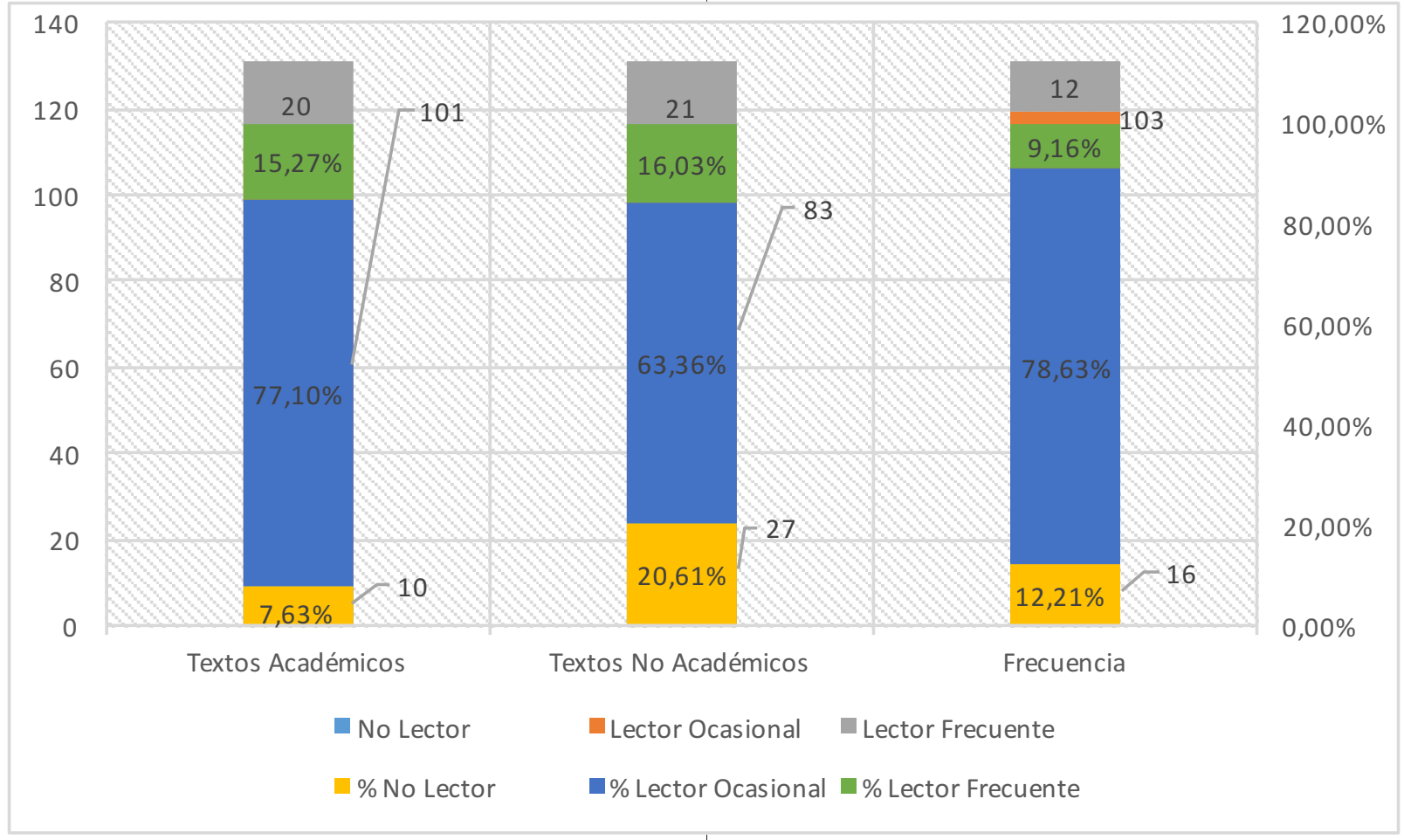

Gráfica 2. Resultados dimensión de frecuencia

Fuente: elaboración propia 
En cuanto a la motivación, un alto porcentaje de estudiantes que leen presenta una motivación extrínseca para leer en inglés (40,5\%), es decir, no leen por iniciativa y gusto propios, sino motivados por el carácter de obligatoriedad de la lectura en inglés en la carrera. Dicho resultado permite observar que una buena parte de los estudiantes de semestres avanzados en el programa de Licenciatura en Bilingüismo no leen materiales en inglés diferentes a los que se exigen en la carrera, y un 3,8\% manifiesta no tener motivación para leer en inglés.

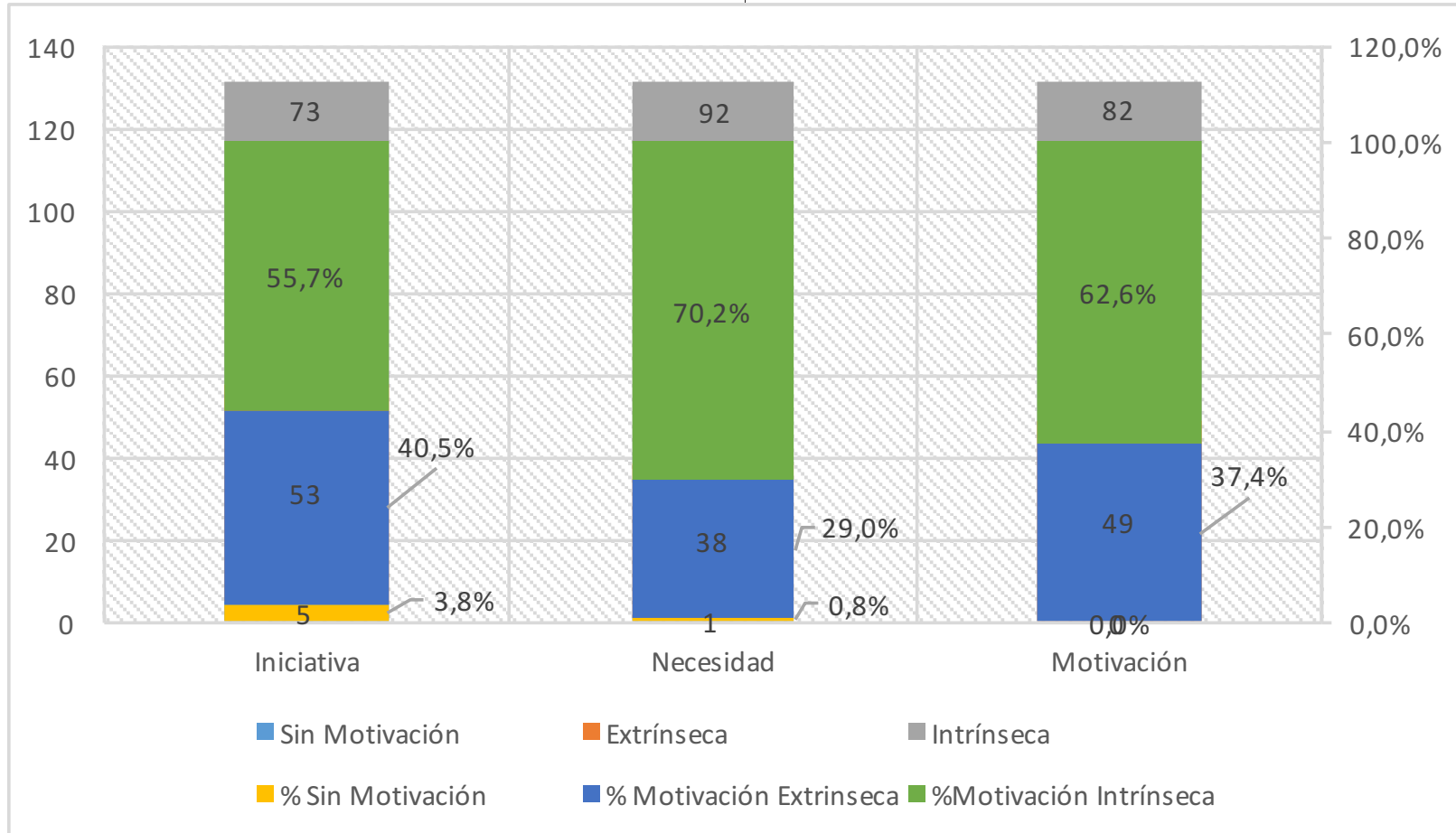

Gráfica 3. Resultados dimensión de motivación

Fuente: Elaboración propia

Este aspecto se pudo constatar en los grupos focales cuando los estudiantes manifestaron :

1. A mí me parece también que es importante leer no solamente libros, sino también documentales o películas porque hay libros que ni siquiera en español me llaman la atención, ahora menos en inglés. Lo considero algo tortuoso en vez de algo agradable, entonces, hay cosas que me gusta más verlas en videos, ver cómo es la gramática y ver cómo se escribe y ver cómo es la pronunciación, eso también es importante. (Estudiante n. ${ }^{07}$. Grupo focal 2, febrero del 2020).
2. La verdad es que no, primordialmente pienso que lo que leo en inglés son memes y cosas así, mas no libros, ni soy literato ni nada por el estilo. (Estudiante n. ${ }^{\circ}$ 1. Grupo focal 1, noviembre del 2019)

3. Pues por lo general, casi no leo en inglés, sino por lo menos documentos cuando me toca presentar alguna actividad o cualquier cosa, pero en mi tiempo libre disfruto hacer otras cosas, menos leer. (Estudiante n. ${ }^{\circ}$ 9. Grupo focal 1 , noviembre del 2019) 
En relación con la autopercepción, si se atiende a la gráfica 4 , un $72,5 \%$ de los estudiantes se considera competente a la hora de comprender los textos en inglés. En cuanto a la fluidez, se evidencia que un $63,4 \%$ se percibe como principiante, es decir, que necesitan de varias lecturas para alcanzar la comprensión y la velocidad de lectura es lenta. El 58,0 $\%$ se autopercibe capaz de leer textos en inglés de distinta complejidad, aun cuando les lleve tiempo hacerlo.

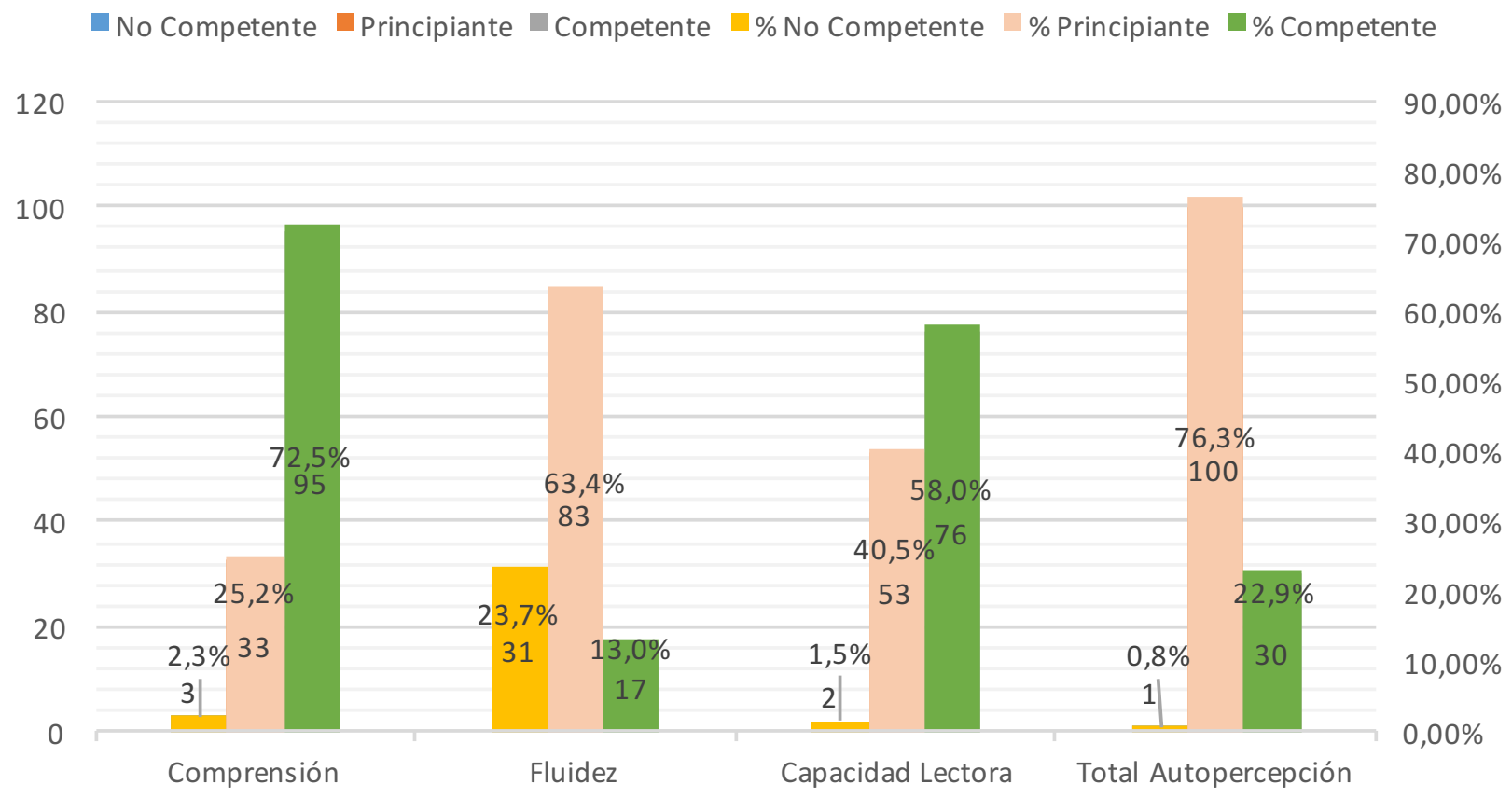

Gráfica 4. Resultados dimensión de autopercepción

Fuente: elaboración propia

Luego de analizar la actitud, la frecuencia y la motivación de los estudiantes del programa de Licenciatura en Bilingüismo, se realizó la caracterización de estos a partir de la clasificación propuesta por Fatiloro et al. (2017), expuesta más arriba, y teniendo en cuenta también los aportes de la literatura revisada:

a. Heavy readers: estudiantes con una actitud positiva, leen frecuentemente y presentan una motivación intrínseca para leer en inglés.

b. Moderate readers: estudiantes con una actitud positiva, leen ocasionalmente en inglés y por una motivación intrínseca. c. Dormant readers: estudiantes con una buena actitud frente a la lectura en inglés, pero que leen ocasionalmente por motivación extrínseca, es decir, que designan un tiempo para leer en inglés solo por cumplir con algún factor externo,

d. Unmotivated non readers: estudiantes con una actitud negativa frente a la lectura en inglés, nunca leen y, si lo hacen ocasionalmente, es por una motivación extrínseca, es decir, nunca por iniciativa o gusto propios.

En la tabla 6 se evidencia el porcentaje de estudiantes que corresponde a cada categoría 
Table 3. Clasificación de los lectores

\begin{tabular}{|c|c|c|}
\hline Categoria & Frecuencia & Porcentaje (\%) \\
\hline Heavy reader & 9 & 7 \\
\hline Moderate reader & 47 & 36 \\
\hline Dormant reader & 7 & 5 \\
\hline Unmotivated & 39 & 30 \\
\hline Falsos lectores & 29 & 22 \\
\hline Total & 131 & 100 \\
\hline
\end{tabular}

Ahora bien, los resultados arrojados por el test de autorreporte mostraron un comportamiento particular en algunos casos, lo que no permite clasificarlos en ninguna de las cuatro categorías mencionadas. Las respuestas de varios estudiantes (22\%) fueron contradictorias en las diferentes dimensiones del test como, por ejemplo, aquellos que presentan una actitud negativa frente a la lectura en inglés, no leen nunca en inglés por interés propio, pero dicen tener una motivación intrínseca para leer. Estas contradicciones dan cuenta de un fenómeno particular que Larrañaga y Yubero (2005) han identificado durante sus quince años de experiencia en investigación sobre hábito lector: el fenómeno de los falsos lectores. Los autores acuñaron este término para referirse a aquellos sujetos que pretenden dar una mejor imagen como lectores, pero que en realidad no leen de forma voluntaria.

Esta categoría nació ante la necesidad de explicar lo que ocurría con aquellos estudiantes que arrojaban resultados contradictorios en los instrumentos de medición, es decir, aquellos que aparecen como lectores en una variable y como no lectores en otra. Según los autores, estos sujetos estarían distorsionando su imagen como lectores para potenciar una valoración positiva. Los falsos lectores leen algunas veces, pero no incorporan la lectura a su estilo de vida; es más, esta se encuentra en último lugar cuando de actividades de tiempo libre se trata. En otros términos, se autorreconocen como lectores, pero realmente no tienen hábito lector (Yubero et al., 2014). La categoría de falsos lectores está influenciada por la deseabilidad social, es decir, es provocada por la necesidad de los estudiantes de responder a la imagen positiva que la sociedad ha construido sobre la lectura (Larrañaga y Yubero, 2019).

Esta situación de falsos lectores se pudo constatar en los grupos focales realizados con los estudiantes de Licenciatura en Bilingüismo, donde se evidenciaron algunas ambivalencias durante el diálogo:

1. Yo disfruto la lectura en inglés, pero, como digo, no lo hago muy seguido; pero igual, sí me agrada porque eso te ayuda a ampliar tu vocabulario, lo disfruto bastante. (Estudiante n. ${ }^{\circ}$ 11. Grupo focal 1, noviembre del 2019).

2. Realmente me gusta, pero no lo tengo como un hábito. Actualmente lo que leo es respecto a la universidad, sobre todo, en la materia de 
legislación que necesita mucha lectura, los decretos las leyes, pero de lectura propia que me llame la atención, actualmente no lo hago. (Estudiante n. ${ }^{\circ}$ 4. Grupo focal 1, noviembre del 2019).

Por otro lado, debido a que el hábito de lectura es individual, social y culturalmente construido, no es de extrañar que la influencia de los padres pueda incidir en el comportamiento lector de los estudiantes. Cuando los padres animan a sus hijos a leer, los niños y jóvenes aprecian el valor de esta actividad y confían en su competencia para la lectura (Iftanti, 2015; Xi et al., 2019). Al respecto, los estudiantes que se consideran lectores dejaron saber en los grupos focales la importancia del acompañamiento de familiares, profesores o padres en el fortalecimiento de los hábitos de lectura en general:

3. Mi papá es uno de los pioneros que siempre me incentiva a leer, de hecho, desde pequeña siempre ha sido un hábito que él ha querido infundir en sus hijas y que lo ha hecho y lo sigue haciendo. (Estudiante n. ${ }^{\circ} 7$. Grupo focal 1, noviembre del 2019).

4. A mí me miran mis papás; siempre me dicen que uno debe leer, así sean dibujos animados, ellos dicen que no terminaron sus estudios, pero quieren que uno siempre esté actualizado. (Estudiante n. ${ }^{\circ}$ 8. Grupo focal 2, febrero del 2020).

\section{Discusión y conclusiones}

Con los resultados obtenidos, se observa que los estudiantes presentan una actitud positiva hacia la utilidad de la lectura en inglés, aunque luego manifiestan que suelen leer poco. En primera instancia, estos resultados podrían parecer llamativos; no obstante, se han hallado resultados similares en otras investigaciones. Ello se debe a que los estudiantes, en general, suelen reconocer la importancia y la utilidad de la lectura desde la perspectiva de la exigencia o del deseo social, especialmente en una carrera de formación docente.

El reconocimiento de la utilidad y la importancia de la lectura en inglés para su formación académica y ejercicio profesional no implica necesariamente que los estudiantes tengan un gusto genuino por esta o que decidan leer en inglés por una motivación intrínseca. Ryan y Deci (2000), autores de la teoría de la autodeterminación (TAD), consideran la motivación intrínseca como "la manifestación prototípica de la tendencia humana hacia el aprendizaje y la creatividad" (p. 2). Si se traslada esto al terreno del hábito lector, se esperaría que un estudiante de un programa de bilingüismo presentara una motivación intrínseca hacia la lectura en inglés, pero dado que la naturaleza humana no es unívoca, se debe aceptar que no todos los estudiantes responden de la misma manera a las experiencias o factores del entorno. Así, como expresan Mezzalira y Boruchovitch (2016), el comportamiento lector se sitúa en algún punto del continuum motivacional (señalado por Ryan y Deci) que se caracteriza por mayor o menor autonomía, sentido de competencia y autodeterminación: leer para ganar alguna recompensa o evitar un castigo; leer para demostrar al profesor que se es un buen alumno; leer porque es importante para tener éxito en los estudios, leer por placer, etc.

Los datos arrojados por el test y lo expresado por los estudiantes en los grupos focales evidencian porcentajes bajos en cuanto a la actitud, la frecuencia y la motivación intrínseca hacia la lectura en inglés. Este hecho no se puede soslayar si se tiene en cuenta que estos estudiantes se están formando para ser docentes en inglés, de modo que idealmente deberían tener una buena disposición hacia la lectura en lengua inglesa. La situación descrita puede deberse a deficiencias en el nivel de inglés de los estudiantes, que les impide comprender de manera adecuada el material bibliográfico, lo que a su vez produce una percepción de inseguridad frente al dominio del idioma que los lleva a evitar las actividades de lectura. Como ya se mencionó, una buena parte de los estudiantes del programa se agota fácilmente o siente estrés al leer en inglés.

Luego del análisis estadístico sobre los hábitos de lectura en inglés de los estudiantes de los niveles de formación básico profesional y profesional, se puede concluir que se hace necesario el diseño de un plan 
de acción que desde el currículo y las actividades extracurriculares permita fomentar actitudes más positivas hacia la lectura en inglés de los estudiantes, y una mayor práctica lectora en inglés para afianzar los hábitos de lectura en la lengua extranjera.

\section{Estrategias preliminares para fomentar el hábito lector en inglés}

Se esperaría que la lectura en inglés fuera una de las actividades prioritarias entre los estudiantes de licenciatura en lenguas extranjeras o bilingüismo para poder acceder a múltiples materiales, medios y recursos, o para mantener un contacto natural con la lengua extranjera. No obstante, cuando eso no ocurre, se requiere el desarrollo de actividades significativas que afiancen la motivación de los estudiantes para mejorar las competencias lectoras en inglés, o el desarrollo de planes institucionales que conduzcan a repensar el rol que los actores de la comunidad académica desempeñan en el fortalecimiento del bilingüismo. Fomentar el hábito lector en inglés entre los estudiantes requiere de acciones pedagógicas que posibiliten el acercamiento a la lectura desde el interés y no solo desde instancias evaluativas.

Los docentes que consideran solo el valor instrumental de la lectura no pueden llegar a formar lectores, es necesario entonces ir más allá de la instrumentalidad curricular y de obligatoriedad de la lectura, y tratar de insertar el comportamiento lector como actividad placentera que se puede desarrollar en tiempos de ocio (Yubero et al., 2014). Partiendo de esta premisa, y atendiendo a lo expresado por los estudiantes en los grupos focales, se contemplará la apertura de un club de lectura en inglés que permita a los estudiantes del programa de Licenciatura en Bilingüismo leer textos académicos y no académicos en el marco de una interacción lúdica y entre pares. Si bien habrá acompañamiento de los docentes del área, este club deberá estar liderado por un estudiante, quien, junto con sus compañeros, determinará las lecturas por hacer y las dinámicas de diálogo. De igual manera, se propone retomar una asignatura electiva de literatura en inglés, que permita a los estudiantes apropiarse de otros estilos de escritura atendiendo a aspectos estéticos y discursivos distintos.

Por otra parte, para fortalecer la integración de la lectura en el currículo, cabe señalar la importancia de incorporar enfoques significativos como el de extensive reading para fomentar de manera transversalizada el hábito lector en inglés. Es decir, es necesario que desde todas las asignaturas de los niveles de formación básico y básico-profesional se destinen espacios de la clase que propicien la lectura de interés personal y que posibiliten el diálogo abierto sobre lo leído:

In ER [Extensive Reading] students read large quantities of easy material in the foreign language. They read for information and enjoyment, with the primary goal of achieving a general, overall meaning of the reading material. Students select their own reading material and are encouraged to stop reading if it is not interesting or too difficult; over time, they are also encouraged to expand their reading comfort zone the range of material that can be read easily and with confidence. (Day, 2013, pp. 10-11)

De acuerdo con Day y Bamford (1998), en la enseñanza del inglés como segunda lengua se encuentran, por lo general, estudiantes que son renuentes a la lectura en ese idioma y que expresan temor ante ello y no suelen leer materiales diferentes a los asignados por el docente. En este contexto, el enfoque de extensive reading adquiere un sentido especial, puesto que ayuda a ganar confianza, fluidez lectora, a ampliar el vocabulario y las habilidades de comprensión; además, permite que los estudiantes se apropien de la segunda lengua y la conviertan en parte de su cotidianidad. La incorporación de este enfoque al programa de Licenciatura en Bilingüismo requiere entonces de un compromiso por parte del cuerpo docente para planificar las formas de abrir estos espacios dentro de la clase, sin que ello represente para los estudiantes una actividad o una tarea más con la que se sientan evaluados.

Las nuevas tecnologías de la información y la comunicación (TIC) también constituyen un medio didáctico, interactivo, agradable, con ambiente 
gráfico atractivo a la mirada que puede incitar a leer mucho más, de manera que las estrategias de fomento de la lectura que logren implementarse deberán contemplar el uso de dichas tecnologías, que permitirán a los estudiantes abordar una multiplicidad de textos cercanos a sus intereses.

En suma, en los programas de licenciatura en idiomas o lenguas extranjeras, un gran número de las actividades académicas desarrolladas involucran prácticas de lectura que requieren de habilidades complejas de pensamiento, por tanto, la lectura se debe practicar con la misma intensidad que en la lengua materna, lo cual supone un esfuerzo por parte de las universidades para generar ambientes de aprendizaje en espacios curriculares y extracurriculares que contribuyan al fortalecimiento del hábito lector en inglés.

\section{Referencias}

Al Nazhari, H., Delfi, S. y Syafri, K. (2016). A study on English reading habits of students of English Study Program of Riau University. Journal Online Mahasiswa (Јом) Bidang Keguruan dan Ilmu Pendidikan, 3(2), 1-8. https://jom.unri.ac.id/index. php/JOMFKIP/issue/view/364

Cárdenas, N. (2018). Perspectivas para un estudio sobre bilingüismo en universidades regionales colombianas. Revista Historia de la Educación Latinoamericana, 20(31), 125-142. 10.19053/01227238.8566

Day, R. y Bamford, J. (1998). Extensive reading in the second language classroom. Cambridge University Press.

Day, R. (2013). Creating a successful extensive reading program. Tesl Reporter, 46(1y2), 10-20.

Fatiloro, F., Adesola, O., Hameed, B. y Adewumi, O. (2017). A survey on the reading habits among colleges of education students in the information age. Journal of Education and Practice, 8(8), 106-110.

Gómez, A. (2014). Los hábitos lectores en inglés de futuros maestros: Implicaciones didácticas. Investigaciones sobre Lectura, 2, 24-31.

Iftanti, E. (2012). A survey of the English Reading Habits of EFL Students in Indonesia. TEFLIN Journal, 23(2), 149-164.
Iftanti, E. (2015). What makes EFL students establish good reading habits in English. International Journal of Education and Research, 3(5), 365-374.

Larrañaga, M. y Yubero, S. (2005). El hábito lector como actitud: El origen de la categoría de "falsos lectores". Ocnos, 1, 43-60.

Larrañaga, M. y Yubero, S. (2019). La compleja relación de los docentes con la lectura: El comportamiento lector del profesorado de Educación Infantil y Primaria en formación. Bordón, 71(1), 31-45.

Ministerio de Educación, Cultura y Deporte de España. (2001). Hábitos de lectura de los adolescentes españoles. Secretaría General de Educación y Formación Profesional.

Ministerio de Educación Nacional. (2006). Estándares Básicos de Competencias en Lenguas Extranjeras: Inglés.

Ministerio de Educación Nacional. (2017). Resolución 18583 de 2017, "Por la cual se ajustan las características específicas de calidad de los programas de Licenciatura para la obtención, renovación o modificación del registro calificado, y se deroga la Resolución 2041 de 2016". https://www.usbcali.edu. co/sites/default/files/resolucion_final_18583_de_ 2017deroga_2041.pdf

Mezzalira, M. y Boruchovitch, E. (2016). Escala de Motivação para a Leitura para Adolescentes e Jovens: Propriedades Psicométricas. Psicologia: Teoria e Pesquisa, 32(2), 1-9.

Rajab, H. y Al-Sadi. (2015). An empirical study of reading habits and interests of Saudi University EFL Learners. International Journal of Linguistics, 7(2), 1-17.

Ryan, R. y Deci, E. (2000). La teoría de la autodeterminación y la facilitación de la motivación intrínseca, el desarrollo social y el bienestar. The American Psychological Association, 55(1), 68-78.

Xia, T., Gu, H. y Li, W. (2019). Effect of Parents' Encouragement on Reading Motivation: The Mediating Effect of Reading Self-Concept and the Moderating Effect of Gender. Frontiers of Psychology, 10, 1-8. https://doi.org/10.3389/fpsyg.2019.00609

Yubero, S., Larrañaga, E. y Pires, N. (2014). Estudo sobre os hábitos de leitura dos estudantes portugueses do ensino superior. Instituto Politécnico de Castelo Branco. 
Universidad Pedagógica Nacional

Facultad de Humanidades

\section{Anexo}

\section{Test de autorreporte}

\section{Licenciatura en Bilingüismo con énfasis en Inglés}

\section{Proyecto de investigación}

Hábitos de lectura en inglés de los estudiantes a partir del nivel de formación básico profesional de la Licenciatura en Bilingüismo con énfasis en Inglés de Unicolombo.

\section{Test de autorreporte}

\section{Caracterización de la población}

\begin{tabular}{l|l|l|l}
\hline Edad & Género & $\begin{array}{l}\text { Nivel de inglés } \\
\text { (según último APTIS) }\end{array}$ & Semestre \\
\hline Código: & ¿Trabaja actualmente como docente? & En primaria ___ En secundaria _ _ _ _ _ & \\
\hline
\end{tabular}

Por favor, marque con una $\mathrm{X}$ su respuesta.
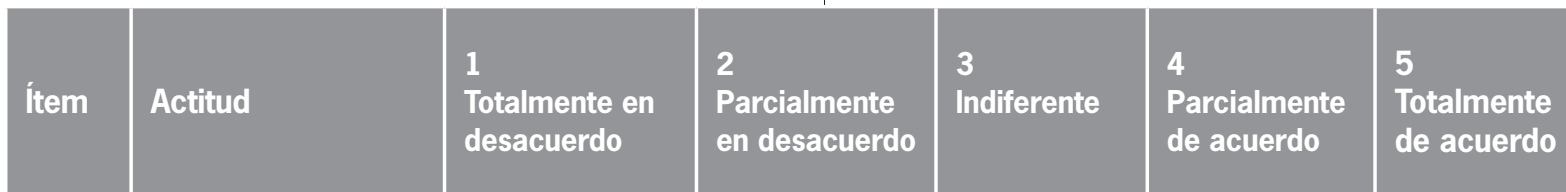
A1 Leer en inglés me agota fácilmente.
A2 Me llama poco la atención leer en inglés
A3 Cuando leo textos extensos en inglés me pongo ansioso.
La lectura en inglés de textos de entretenimiento como
A4 revistas de farándula, historietas, carica- turas, etc. es poco atractiva para mí.




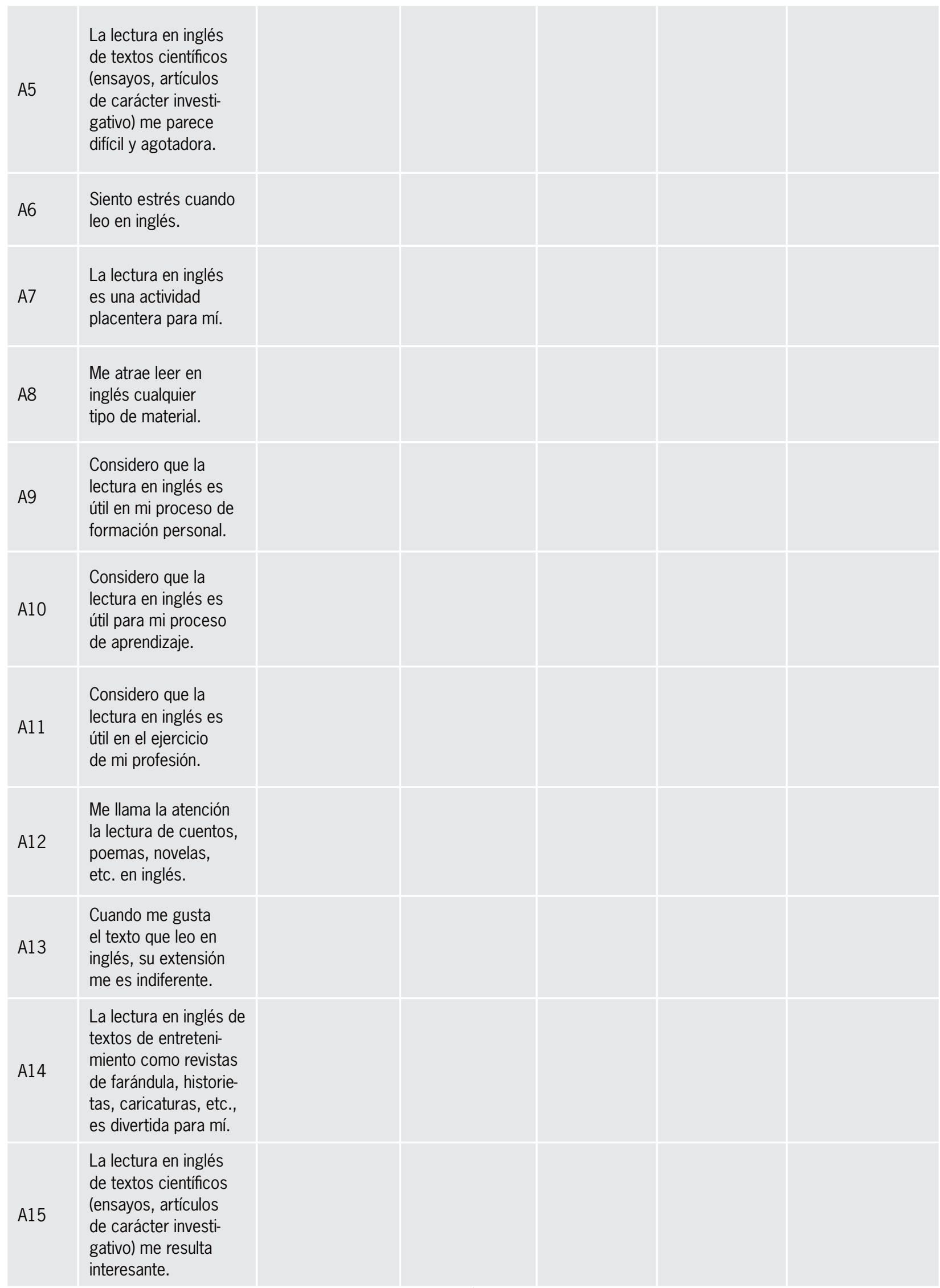


Universidad Pedagógica Nacional

Facultad de Humanidades

\begin{tabular}{|c|c|}
\hline Ítem & Frecuencia \\
\hline $\mathrm{F} 1$ & $\begin{array}{l}\text { Leo libros acadé- } \\
\text { micos en inglés. }\end{array}$ \\
\hline F2 & $\begin{array}{l}\text { Leo blogs y páginas } \\
\text { web académicos } \\
\text { en inglés. }\end{array}$ \\
\hline F3 & $\begin{array}{l}\text { Leo capítulos de libros } \\
\text { académicos en inglés. }\end{array}$ \\
\hline F4 & $\begin{array}{l}\text { Leo artículos cien- } \\
\text { tíficos en inglés. }\end{array}$ \\
\hline F5 & $\begin{array}{l}\text { Leo novelas y textos } \\
\text { literarios en inglés. }\end{array}$ \\
\hline F6 & $\begin{array}{l}\text { Leo blogs y páginas } \\
\text { web no académi- } \\
\text { cos en inglés. }\end{array}$ \\
\hline F7 & $\begin{array}{l}\text { Leo revistas de entre- } \\
\text { tenimiento en inglés. }\end{array}$ \\
\hline F8 & $\begin{array}{l}\text { Leo libros de au- } \\
\text { toayuda en inglés. }\end{array}$ \\
\hline F9 & $\begin{array}{l}\text { Leo cómics y cari- } \\
\text { caturas en inglés. }\end{array}$ \\
\hline F10 & $\begin{array}{l}\text { Leo periódicos } \\
\text { en inglés. }\end{array}$ \\
\hline
\end{tabular}
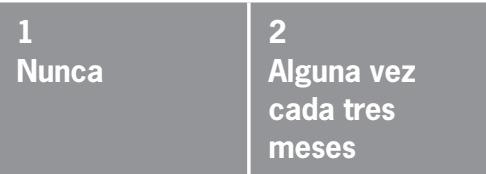

\begin{tabular}{|l|}
\hline 3 \\
Alguna vez \\
al mes
\end{tabular}
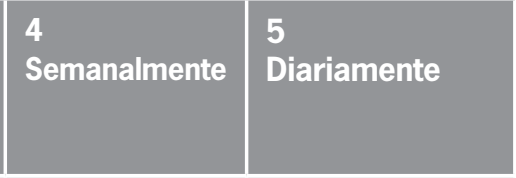

(n)
meses

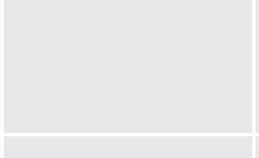

$x^{2}+x^{2}$

.




\begin{tabular}{|c|c|c|c|c|c|c|}
\hline M4 & $\begin{array}{l}\text { Leo en inglés para } \\
\text { estar informado(a) de } \\
\text { los avances e innova- } \\
\text { ciones relacionados } \\
\text { con mi disciplina. }\end{array}$ & & & & & \\
\hline M5 & $\begin{array}{l}\text { Leo textos en } \\
\text { inglés por interés } \\
\text { y gusto propio. }\end{array}$ & & & & & \\
\hline M6 & $\begin{array}{l}\text { Leo en inglés porque } \\
\text { mejora mi compe- } \\
\text { tencia lectora. }\end{array}$ & & & & & \\
\hline M7 & $\begin{array}{l}\text { Leo en inglés porque } \\
\text { siempre aprendo } \\
\text { nuevas palabras. }\end{array}$ & & & & & \\
\hline M8 & $\begin{array}{l}\text { Leo en inglés porque } \\
\text { quiero conectarme } \\
\text { con nuevas expe- } \\
\text { riencias culturales. }\end{array}$ & & & & & \\
\hline M9 & $\begin{array}{l}\text { Leo en inglés materia- } \\
\text { les complementarios } \\
\text { a los de la clase por } \\
\text { iniciativa propia. }\end{array}$ & & & & & \\
\hline M10 & $\begin{array}{l}\text { Prefiero leer en inglés } \\
\text { antes que realizar } \\
\text { otras actividades } \\
\text { en mi tiempo libre. }\end{array}$ & & & & & \\
\hline M11 & $\begin{array}{l}\text { Leo en inglés porque } \\
\text { me gusta el idioma. }\end{array}$ & & & & & \\
\hline litem & Autopercepción & $\begin{array}{l}1 \\
\text { Totalmente en } \\
\text { desacuerdo }\end{array}$ & $\begin{array}{l}2 \\
\text { Parcialmente } \\
\text { en desacuerdo }\end{array}$ & $\begin{array}{l}3 \\
\text { Indiferente }\end{array}$ & $\begin{array}{l}4 \\
\text { Parcialmente } \\
\text { de acuerdo }\end{array}$ & $\begin{array}{l}5 \\
\text { Totalmente } \\
\text { de acuerdo }\end{array}$ \\
\hline AP1 & $\begin{array}{l}\text { Reconozco las ideas } \\
\text { principales cuando leo } \\
\text { un texto en inglés. }\end{array}$ & & & & & \\
\hline AP2 & $\begin{array}{l}\text { Infiero la intención co- } \\
\text { municativa de un texto } \\
\text { cuando leo en inglés. }\end{array}$ & & & & & \\
\hline AP3 & $\begin{array}{l}\text { Me es fácil identificar } \\
\text { el punto de vista del } \\
\text { autor cuando leo } \\
\text { un texto inglés. }\end{array}$ & & & & & \\
\hline AP4 & $\begin{array}{l}\text { Considero que debo } \\
\text { mejorar mucho mi } \\
\text { nivel de inglés para } \\
\text { poder leer con fluidez. }\end{array}$ & & & & & \\
\hline
\end{tabular}


Universidad Pedagógica Nacional

Facultad de Humanidades

\begin{tabular}{|l|l|l|||}
\hline AP5 & $\begin{array}{l}\text { Leo en inglés } \\
\text { con fluidez. }\end{array}$ \\
\hline AP6 & $\begin{array}{l}\text { Me cuesta leer } \\
\text { textos complica- } \\
\text { dos en inglés. }\end{array}$ \\
\hline AP7 & $\begin{array}{l}\text { Me demoro mucho } \\
\text { cuando leo en inglés. }\end{array}$ & \\
\hline AP8 & $\begin{array}{l}\text { Considero que leo } \\
\text { mejor en inglés } \\
\text { cuando los textos } \\
\text { son sencillos. }\end{array}$ & \\
\hline AP9 & $\begin{array}{l}\text { Considero que } \\
\text { comprendo textos en } \\
\text { inglés, aunque su te- } \\
\text { mática sea compleja. }\end{array}$ & \\
\hline & & \\
\hline
\end{tabular}

¡Gracias por tu colaboración! 